\title{
Burden of Dengue and Chikungunya - A Retrospective Study
}

\author{
J.V. Sathish, Mita D. Wadekar* (1), S. Jayashree and C. Pooja \\ Department of Microbiology, Chamarajanagar Institute of Medical Sciences, Chamarajanagar, Karnataka, India.
}

\begin{abstract}
Arboviral infections like dengue fever and chikungunya are the most common infections that share the same Aedes mosquito vectors. Clinical presentations of these two infections are also similar, especially in initial stages. Non-structural antigen (NS1 Ag)detection for dengue and detection of IgM antibodies by capture ELISA for chikungunya and dengue infection may help in the early diagnosis. Early diagnosis is essential for the treatment and control measures. The present study was conducted to know the burden of dengue and chikungunya. A retrospective study was conducted for a period of 1 year from Dec 2017 to Nov 2018 to know the burden of dengue and chikungunya in Chamarajanagar. Dengue (> 5 days fever) and chikungunya testing was done by IgM antibody capture ELISA kits produced by NIV. Dengue samples ( $<$ days fever) were subjected to NS1 antigen detection by microwell enzymelinked immunosorbent assay (ELISA) from Qualpro diagnostics. The tests were carried out following manufacturer's instruction. Samples received for dengue NS1 Ag testing was 446, of which, 49(11.0\%) were positive and of 730 samples received for IgM antibody, $53(7.3 \%)$ were positive. Age group commonly affected was $\mathbf{0 - 2 0}$ years $44(43.1 \%)$. Of 668 samples received for chikungunya test, 86 (12.9\%) were positive. Maximum number of cases was seen in age group of 21-40 years 45(52.3\%). Males 56(54.9\%) were affected higher than female 46(45.1\%) in dengue infection while in chikungunya, females $45(52.3 \%)$ were more affected than males 41(47.7\%). Both infections are high in the month of June and July. Early detection of dengue by NS1 antigen and detection of IgM antibodies by capture ELISA chikungunya and dengue infection helps in appropriate treatment and initiation of prevention and control measures by community awareness and vector control.

Keywords: Dengue, chikungunya, ELISA
\end{abstract}

*Correspondence: drmdw20@gmail.com

(Received: January 07, 2021; accepted: May 10, 2021)

Citation: Sathish JV, Wadekar MD, Jayashree S, Pooja C. Burden of Dengue and Chikungunya - A Retrospective Study. J Pure Appl Microbiol. 2021;15(2):772-776. doi: 10.22207/JPAM.15.2.30

(C) The Author(s) 2021. Open Access. This article is distributed under the terms of the Creative Commons Attribution 4.0 International License which permits unrestricted use, sharing, distribution, and reproduction in any medium, provided you give appropriate credit to the original author(s) and the source, provide a link to the Creative Commons license, and indicate if changes were made. 


\section{INTRODUCTION}

Among the arboviral infections, dengue fever and chikungunya are most common infections. Dengue virus has four serotypes (DEN-1, DEN-2, DEN-3 and DEN-4). Recently, in 2013 fifth serotype (DEN-5) was discovered from Bangkok. Aedes mosquito is the vector for both these infections. They bite during day time $e^{1,2}$. Dengue affects mainly tropical and subtropical regions of the world. It is prevalent throughout India. Majority of the cases have been reported from Kerala, Tamil Nadu, Karnataka, Orissa, Delhi, Maharashtra and Gujarat ${ }^{3}$. Symptoms of dengue may range from a mild febrile illness to severe illnesses like dengue fever (DF) and dengue hemorrhagic fever (DHF). When a person is infected with any one serotype for the first time develops primary infection. Infection with one serotype of the dengue viruses confers lifelong immunity to that serotype only. Secondary dengue infection occurs in a person infected with second serotype which is different from first serotype and manifests severe form of illness such as DHF due to immune enhancement. ${ }^{4,5}$

Clinical presentations of these two infections are also similar, especially in initial stages characterized by fever, rash, myalgia and arthralgia ${ }^{6}$. As mortality rate and severity is high in dengue fever as compared to chikungunya, all patients are tested only for dengue virus and in rare cases for chikungunya infection. As a result, burden of chikungunya has been missed and also cases go undiagnosed ${ }^{7}$. The environment and man made factors are responsible for the spread of the disease. Poor sanitation, overcrowding, man made breeding sites and climatic changes favors infection. High temperature and high humidity prolongs the life span and spread of the vector. ${ }^{8-10}$

Based on clinical presentation diagnosis of dengue and chikungunya is difficult. Although, majority of the infections are self-limiting, timely diagnosis of dengue helps in appropriate management in severe cases. ${ }^{11}$ Enzyme-linked immunosorbent assays (ELISAs), RT-PCR and virus isolation helps in diagnosis of these infections. ELISA detect both immunoglobulin (Ig) M and IgG antibodies from samples. Detection of dengue non-structural antigen (NS1 Ag) may help in the early diagnosis and treatment of dengue ${ }^{12,13}$. Early diagnosis is essential for the early and appropriate treatment and also for implementation of control measures. The present study is conducted to know the burden of dengue and chikungunya.

\section{MATERIALS AND METHODS}

Retrospective study was conducted at Microbiology laboratory, District hospital, Chamarajanagar Institute of Medical Sciences for duration of 1 year from July 2018 to June 2019. Clinically suspected cases of dengue and chikungunya were included and other were excluded. Related data like age, gender and results are collected from Laboratory registers. Serum samples from these cases were collected. Total of 446 samples were received for dengue NS1 Ag testing, 730 samples for dengue IgM antibody and 668 samples for chikungunya test.

Dengue ( $>5$ days fever) and chikungunya testing was done by IgM antibody capture ELISA kits produced by NIV. Dengue samples ( $<5$ days fever) were subjected to dengue NS1 antigen detection by microwell enzyme-linked immunosorbent assay (ELISA) from Qualpro diagnostics (A division of Tulip Diagnostics (P), Verna, Goa, India). The tests were carried out following manufacturer's instruction. Data analysis was done using MS Excel.

\section{Ethical clearance}

Ethical clearance was obtained from the Institutional Ethical clearance committee of Chamarajanagar Institute of medical sciences, Chamarajanagar.

\section{RESULTS}

Samples received for dengue NS1 Ag testing was 446 , of which, $49(11.0 \%)$ were positive and of 730 samples received for IgM antibody, 53 (7.3\%) were positive. (Table 1) shows prevalence of dengue infection by NS1 Ag detection and by IgM antibody dectection. (Fig. 1) shows that, of 668 samples received for chikungunya testing, $86(12.9 \%)$ were positive and $582(87.1 \%)$ were

Table 1. Prevalence of dengue infection

\begin{tabular}{lcc}
\hline \multirow{2}{*}{ Samples } & \multicolumn{2}{c}{ Dengue No. (\%) } \\
\cline { 2 - 3 } & NS1 Ag & IgM \\
\hline Positive samples & $49(11.0)$ & $53(7.3)$ \\
Negative samples & $397(89.0)$ & $677(92.7)$ \\
Total & $446(100)$ & $730(100)$ \\
\hline 3
\end{tabular}


negative. Age group commonly affected for dengue infection was $0-20$ years $44(43.1 \%)$ and for chikungunya was $21-40$ years $45(52.3 \%)$ than any other age group which is shown in (Table 2).(Table 2) shows that males $56(54.9 \%)$ were affected more than female $46(45.1 \%)$ in dengue infection while in chikungunya, females $45(52.3 \%)$ were more affected than males $41(47.7 \%)$. (Table 4 ) shows monthly distribution of dengue and chikungunya infections. Maximum cases of dengue were detected in the month of June and July i.e $16.3 \%$ and $20.4 \%$ by NS1 antigen and $22.7 \%$ and $28.3 \%$ by IgM antibody respectively. Chikungunya was peak in the month of June (22.1\%), July (18.6\%) and August (11.6\%).

Table 2. Age wise distribution of dengue and chikungunya cases

\begin{tabular}{lccc}
\hline \begin{tabular}{l} 
Age group $\begin{array}{l}\text { (years) } \\
\end{array}$ \\
\cline { 2 - 3 }
\end{tabular} & \multicolumn{2}{c}{$\begin{array}{c}\text { Dengue } \\
\text { NO. (\%) }\end{array}$} & $\begin{array}{c}\text { Chikungunya } \\
\text { NO. (\%) }\end{array}$ \\
\cline { 2 - 3 } NS1 Ag & \multicolumn{1}{c}{ IgM } & \\
\hline $0-20$ & $20(40.8)$ & $24(45.3)$ & $26(30.3)$ \\
$21-40$ & $24(49.0)$ & $17(32.1)$ & $45(52.3)$ \\
$41-60$ & $02(4.1)$ & $10(18.8)$ & $13(15.1)$ \\
$>60$ & $03(6.1)$ & $02(3.8)$ & $02(2.3)$ \\
Total & $49(100)$ & $53(100)$ & $86(100)$ \\
\hline
\end{tabular}

Table 3. Gender wise distribution of Dengue and Chikungunya cases

\begin{tabular}{lccc}
\hline Gender & \multicolumn{2}{c}{$\begin{array}{c}\text { Dengue } \\
\text { NO. (\%) }\end{array}$} & $\begin{array}{c}\text { Chikungunya } \\
\text { NO. (\%) }\end{array}$ \\
\cline { 2 - 3 } & NS1 Ag & IgM & \\
\hline Male & $26(53.1)$ & $30(56.6)$ & $41(47.7)$ \\
Female & $23(46.9)$ & $23(43.4)$ & $45(52.3)$ \\
Total & $49(100)$ & $53(100)$ & $86(100)$ \\
\hline
\end{tabular}

\section{DISCUSSION}

Dengue virus belongs to a genus Flavivirus and chikungunya to an Alphavirus. ${ }^{14}$ These arboviral infections, transmitted by Aedes Aegypti mosquito are of great concern. Both these viruses may cocirculate and can be transmitted together. ${ }^{15}$ Changes in the genotype and mutations in the genome have been detected for both dengue and chikungunya viruses ${ }^{14}$. Appropriate management of patient requires accurate and early diagnosis of infection.

In present study, dengue prevalence was $11 \%$ by NS1 antigen detection which is similar to study done by Nissi Mathew et al. which showed $11.6 \%$ prevalence ${ }^{16}$. Prevalence of dengue infection by detection of IgM antibody in our study was $7.3 \%$ and study done by Nepal H.P et al. showed $8.5 \% \cdot{ }^{17}$ Our study showed prevalence of chikungunya as $12.7 \%$ and study done by Ms. Akanksha Tomar et al. showed $16 \%{ }^{18}$. Before

Table 4. Monthly distribution of Dengue and Chikungunya cases

\begin{tabular}{lccc}
\hline Month & $\begin{array}{c}\text { Dengue } \\
\text { NS1 } \\
\text { No. (\%) }\end{array}$ & $\begin{array}{c}\text { Dengue } \\
\text { IgM } \\
\text { No. (\%) }\end{array}$ & $\begin{array}{c}\text { Chikungunya } \\
\text { IgM } \\
\text { No. (\%) }\end{array}$ \\
\hline July & $10(20.4)$ & $15(28.3)$ & $16(18.6)$ \\
August & $02(4.1)$ & $05(9.4)$ & $10(11.6)$ \\
September & $04(8.2)$ & $02(3.8)$ & $06(7.0)$ \\
October & $01(2.0)$ & $00(00)$ & $01(1.2)$ \\
November & $01(2.0)$ & $01(1.9)$ & $02(2.3)$ \\
December & $02(4.1)$ & $02(3.8)$ & $04(4.7)$ \\
January & $05(10.2)$ & $03(5.7)$ & $05(5.8)$ \\
February & $02(4.1)$ & $02(3.8)$ & $07(8.1)$ \\
March & $03(6.1)$ & $04(7.6)$ & $05(5.8)$ \\
April & $05(10.2)$ & $02(3.8)$ & $08(9.3)$ \\
May & $06(12.3)$ & $05(9.4)$ & $03(3.5)$ \\
June & $08(16.3)$ & $12(22.7)$ & $19(22.1)$ \\
Total & $49(100)$ & $53(100)$ & $86(100)$ \\
\hline
\end{tabular}

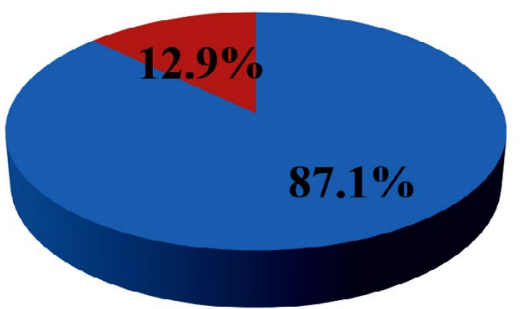

\section{Negative}

- Positive

Fig. 1. Prevalence of chikungunya infection 
antibodies appear, NS1 antigen detection is helpful for early and rapid detection of infection ${ }^{19}$. Chikungunya is commonly associated with mild to moderate infection but dengue can cause severe complications ${ }^{20}$. Early diagnosis of dengue infection by NS1 antigen detection and appropriate treatment is required to prevent complications.

Dengue detection by NS1 antigen and by IgM antibody detection were commonly seen in the age group of $21-40$ years (49\%) and $0-20$ $(45.3 \%)$ respectively. Chikungunya was commonly seen in the age group of 21-40 years (52.3\%). Study done by Abhishek KS et al showed similar age group involvement ${ }^{21}$. Present study showed males were commonly affected in dengue i.e(53.1\%) by NS1 antigen and ( $56.6 \%)$ by IgM antibody which correlates with the study done by R. Ganesan et al. who reported $51.9 \%$. In chikungunya, females $(52.3 \%)$ were commonly affected than males (47.7\%). Study conducted by Cueva J.T.D et al. showed $68.9 \%$ of the 777 confirmed cases of chikungunya were females 23 . Gender differences are common due to community-specific habits, customs or behaviours ${ }^{24}$. Maximum cases of dengue were seen in the month of June and July and chikungunya in the month of June, July and August which is similar to the study done by Karthik et $\mathrm{a}^{25}$. Most of the studies showed similar seasonal distribution. As humid conditions increases life span of vector, transmission occurs at the beginning of rainy season ${ }^{1}$.

Serological tests are the most commonly used diagnostic method. But these tests cannot identify the serotype causing infection. Polymerase chain reaction (PCR) is becoming the rapid detection method and also can be used for detection of serotypes and quantification of viral load $^{26}$. Diagnosis of dengue and chikungunya becomes difficult without adequate serological and other diagnostic tests. Seroprevalence studies are needed to know the prevalence of these infections in particular areas, to prevent transmission of the disease and to implement effective control measures.

\section{CONCLUSION}

Dengue and chikungunya infection exists in our set up. Detection of NS1 antigen and IgM antibodies by capture ELISA helps to know the etiology and also early and rapid diagnosis helps in appropriate treatment. Prevention and control measures can be initiated by community awareness and vector control.

\section{ACKNOWLEDGMENTS}

None.

\section{CONFLICT OF INTEREST}

The authors declare that there is no conflict of interest.

\section{AUTHORS' CONTRIBUTION}

MDW drafted the manuscript, compiled data and designed figures and tables. JS and PC collected data. SJV supervised and reviewed the manuscript.

\section{FUNDING}

None.

\section{DATA AVAILABILITY}

All datasets generated or analyzed during this study are included in the manuscript.

\section{ETHICS STATEMENT}

This article does not contain any studies with human participants or animals performed by any of the authors.

\section{REFERENCES}

1. Sudhan SS, Sharma M, Sharma P, Gupta RK, Sambyal SS, Sharma S. Serosurveillance of Dengue, Chikungunya and Zika in Jammu, a Sub-Himalayan Region of India. J Clin Diagn Res. 2017;11(11): DC05-DC08.doi: 10.7860/ JCDR/2017/29210.10848

2. Kalawat U, Sharma KK, Reddy SG. Prevalence of dengue and chickungunya fever and their co-infection. Indian J Pathol Microbiol. 2011; 54(4): 844-846. doi: 10.4103/0377-4929.91518

3. Modi KP, Patel DA, Vegad MM, Mistry AU, Padariay NJ, Rathod AB. Sero-Prevalence of Dengue and Chikungunya, their Co-Infection and Seasonal Trends of These Infections at a Tertiary Care Hospital, Ahmedabad, Gujarat. Int J Microbiol Res. 2017;9(1):819-822.

4. Nguyen THT, Clapham HE, Phung KL, et al. Methods to discriminate primary fromsecondary dengue during acutesymptomatic infection. BMC Infectious Diseases. 2018;18:375. doi: 10.1186/s12879-018-3274-7

5. Khurram M, Qayyum W, Hassan SJ, Mumtaz S, Bushra $\mathrm{HT}$, Umar M. Dengue hemorrhagic fever: Comparison of patients with primary and secondary infections. Journal of Infection and Public Health. 2014;7(6):489495. doi: 10.1016/j.jiph.2014.05.005

6. Shrihari N, Kumudini TS, Mariraj J, Krishna S. The 
Prevalence of Arboviral diseases mainly Dengue, Chikungunya and Japanese B Encephalitis in and around Bellary district, Karnataka. J Pharm Biomed Sci. 2012;15(10):1-3.

7. Dinkar A, Singh J, Prakash P, Das A, Nath G. Hidden burden of chikungunya in North India; A prospective study in a tertiary care centre. $J$ Infect Public Health. 2017;11(4):586-591. doi: 10.1016/j.jiph.2017.09.008

8. Bhagwati C, M M, Mehta KD, Y S G. Profile of The Chikungunya Infection: A Neglected Vector Borne Disease which is Prevalent In The Rajkot District. J Clin Diagn Res. 2013;7(6):1008-1011. doi:10.7860/ JCDR/2013/5307.3057

9. Selvakumari S. A study on detection of prevalence of dengue, chikungunya, leptospirosis and their coinfection in acute febrile patients. University Journal of Pre and Para Clinical Sciences. 2018;4(1).

10. Chandran R, Azeez PA. Outbreak of dengue in Tamil Nadu, India. Current Science. 2015;109(1):171-176.

11. Kajeguka DC, Kaaya RD, Mwakalinga S, et al. Prevalence of dengue and chikungunyavirus infections in northeastern Tanzania: a cross sectional study among participants presenting with malaria-like symptoms. BMC Infectious Diseases. 2016;16:183. doi: 10.1186/ s12879-016-1511-5

12. Lertanekawattana S, Anantapreecha S, Jiraphongsa $C$, et al. Prevalence and characteristics of dengue and chikungunya infections among acute febrile patients in NongKhaiProvince, Thailand. Southeast Asian J Trop Med Public Health. 2013;44(5):780-790.

13. Deeba F, Afreen N, Islam A, et al. Co-infection with Dengue and Chikungunya Viruses. Current Topics in Chikungunya. 2016. doi: 10.5772/64308

14. Cecilia D. Current status of dengue and chikungunya in India. WHO South-East Asia J Public Health. 2014;3(1):22-26. doi: 10.4103/2224-3151.206879

15. Giriraja KV, Pavitra C, Bindumathi PL. Seroprevalence of chikungunya and dengue dual infection and chikungunyamonoinfection among patients with acute febrile illness attending a medical research institute in Bangalore. J Evid Based Med Health. 2017; 4(25):148285. doi: $10.18410 / \mathrm{jebmh} / 2017 / 287$

16. Mathew N, Rajahamsan J, Sahira H, Rani B, Bai RJT. Study on Prevalence of Dengue Fever in a Tertiary Care Hospital, South Kerala. Journal of Medical Science and Clinical Research. 2017;5(1): 15435-15440. doi: $10.18535 / \mathrm{jmscr} / \mathrm{v} 5 \mathrm{i} 1.40$

17. Nepal HP, Ansari S, Gyawali N. Detection of IgM against Dengue Virus in Clinically Suspected Patients Presenting at a Tertiary Care Centre, Narayani Zone, Nepal. J Trop Dis. 2014; 2(3). doi: 10.4172/2329891X.1000139

18. A Tomar, AVB Hodiwala, DD Khiste. Prevalence of Chikungunya Viral Infection in a Tertiary Care Hospital, Navi Mumbai Maharashtra. Journal of Medical Science and Clinical Research. 2017;5(1):15948-15951. doi: 10.18535/jmscr/v5i1.115

19. Manthalkar PS, Peerapur BV. Utility of NS1 Antigen for Diagnosis of Dengue Virus Infection. Journal of Krishna Institute of Medical Sciences University. 2017;6(1):7275.

20. Gandhi BS, Kulkarni K, Godbole M, et al. Dengue and Chikungunya co-infection associated with more severe clinical disease than mono-infection. International $\mathrm{J}$ of Healthcare and Biomedical Research. 2015;3(3):117123.

21. Abhishek KS, Chakravarti A. Simultaneous detection of IgM antibodies against dengue and chikungunya: Coinfection or cross-reactivity? J Family Med Prim Care. 2019;8:2420-2423. doi: 10.4103/jfmpc.jfmpc_365_19

22. Ganesan R, Devamani TSD, Innocent DJP. A Study on the Prevalence of Dengue Virus Infection using NS1 Antigen and IgM Antibody capture ELISA for the Early Diagnosis in and around Madurantakam, India. Int J Curr Microbiol App Sci. 2019;8(02):1596-1600. doi: 10.20546/ijcmas.2019.802.187

23. Cueva JTD, Ples MB, Vitor RJS. Relationship between chikungunya virus prevalence, rainfall, and urbanization in the Philippines. Natl J Physiol Pharm Pharmacol. 2018;8:977-982. doi: 10.5455/ njppp.2018.8.0208204032018

24. NAM Azami, SA Salleh, SA Shah, et al. Emergence of chikungunya sero positivity in healthy Malaysian adults residing in outbreak-free locations: Chikungunya seroprevalence results from the Malaysian Cohort. BMC Infectious Diseases. 2013;13:67. doi: 10.1186/14712334-13-67

25. Karthik R, Vineetha KR, Raut CG, N Shaik, Manjunatha. Seroprevalance of dengue and chikungunya co infection and its clinical correlation in Bangalore city hospitals. International Journal of Current Research. 2014;6(12):11040-11044.

26. SOD Paulal, BALd Fonsecal. Dengue: A Review of the Laboratory Testsa Clinician Must Know to Achieve a Correct Diagnosis. Braz J Infect Dis. 2004;8(6):390-398. doi: 10.1590/S1413-86702004000600002 